\title{
Metaboreflex Activity is Attenuated by Transcutaneous Electrical Nerve Stimulation and Interferential Electrical Stimulation in Healthy Individuals
}

\author{
Julio Zago ${ }^{1}$, Paulo J.C. Vieira², Gerson Cipriano $\mathrm{Jr}^{1}$, André Luis Machado Winter², Marcos D. Silveira²,
} Adriana M. Güntzel Chiappa ${ }^{2}$, Tatiana Z. Rondinel ${ }^{1}$, Francisco Valdez dos Santos ${ }^{1}$, Gaspar R. Chiappa ${ }^{3}$.

${ }^{1}$ Universidade de Brasilia (UnB), Brasilia, Brasil; ${ }^{2}$ Hospital de Clínicas de Porto Alegre, Rio Grande do Sul, Brasil; ${ }^{3}$ Universidade Evangélica de Goiás (UniEVANGELICA), Brasil.

\section{ABSTRACT}

Background: Transcutaneous electrical nervous stimulation (TENS) and interferential electrical stimulation (IES) attenuates muscle metaboreflex by sympathetic nervous modulation. Objective: We tested the hypothesis that IES may be more effective than TENS to improves blood flow which may be linked to greater of deep tissue. Methods: Eleven health subjects were randomized to TENS ( $80 \mathrm{~Hz}, 150 \mu \mathrm{s})$, IES (4000 $\mathrm{Hz}, \triangle \mathrm{AMF}=25 \mathrm{~Hz}$ ) or sham stimulation group, during 30 minutes. The acute intervention was applied on stellate ganglion region (C7-T4). Results: Were measured metaboreflex activity by calf vascular resistance (CVR) and calf blood flow (CBF) and HRV during three times: rest, exercise (static handgrip) and postexercise circulatory occlusion (PECO+ and PECO-). At the exercise peak, compared with TENS and Sham, the IES group reduced CVR $(36 \pm 3$ vs $43 \pm 3 ; p<0.05)$ and increased CBF $(p<0.01)$. Also, IES was associated with a greater reduction on the MMA (IES: $9 \pm 2$, TENS: $14 \pm 4$, Sham: $26 \pm 5$ units; $p<0.01$ ). Furthermore, the IES group had a higher reduction of LF/HF ratio during PECOand PECO+ $(p<0.05)$. Conclusion: The IES over the stellate ganglion region seems to have superior efficacy compared with TENS to attenuate metaboreflex activation and vasodilatory responses during exercise in healthy subjects.

Keywords: Autonomic nervous system; Neuromodulation; Transcutaneous electrical nervous stimulation; Blood flow; Exercise; Heart rate variahilitı

\section{BACKGROUND}

Several studies with application of transcutaneous electrical nerve stimulation (TENS) and interferential electrical stimulation (IES) were recently conducted with special focus on nonanalgesic effects that seem to be related to blood flow effect and vasodilatory mechanisms. ${ }^{(1-3)}$ In this regard, it has been suggested that the application of TENS and IES, low and middle frequency electrical pulses, respectively, over stellate ganglion or peripherally may induce local vasodilation, ${ }^{(1,4-7)}$ attenuating the vascular resistance, that may be linked to improvement of cardiopulmonary adjustment. In addition, these electrical stimulation modalities could also have a favorable impact on the sympathetic nervous system, trough mitigation on the pressor reflex. ${ }^{(8)}$

Considering the variety of the studies methodologies, such as duration, intensity and area under treatment, different physiological responses has been showed, such as, peripheral circulation increase, ${ }^{(9-12)}$ myocardial oxygen increase, and oxygen demand reduction. ${ }^{(13-15)}$ Our research group recently found that TENS applied previously to exercise at stellate ganglion region attenuates muscle metaboreflex activation (reduction in the distribution of muscle blood flow). (2)

This response was linked to an increase in peripheral vasodilatory capacity and reduction of the blood pressure response at the end of the exercise, attenuating sympathetic-mediated vasoconstriction during exercise. In addition, we also tested the effect of the isolated application IES on muscle metaboreflex activity, ${ }^{(3)}$ resulting in significant lower levels of vasoconstrictor tone and marked reduction in muscle metaboreflex activity. However, despite these findings, there is none study that compare these two kinds of electrical stimulation.

In this sense, the aim of the present study was to compare the effectiveness of application of TENS and IES over the ganglionic area and their muscle metaboreflex responses mediated by the autonomic nervous system in healthy individuals. The hypothesis is that the blood pressure, blood flow and resistance vascular response evoked by directly stimulation on ganglion with IES during static exercise would be greater than TENS, due to present higher maximum total current and greater penetration in the tissues, as well as lower accommodation of the stimulated nerve fibers. ${ }^{(1,16)}$

\section{METHODS Patients}

The subjects were 11 healthy volunteered for study participation. All subjects were non-smokers, non-obese and free of any signs or symptoms of disease, as revealed by the medical history, physical examination and electrocardiogram at rest and during exercise. 
The exclusion criteria were use of alcohol or any medication with potential effects on circulatory system. The subjects were instructed not to consume foods or beverages containing caffeine and do not exercise 48 hours before the protocol. Data were collected in Exercise Pathophysiology Research Laboratory and Cardiology Division, Hospital de Clinicas de Porto Alegre, Rio Grande do Sul, Brazil. The procedures were approved by the Institutional Review Board of the Hospital de Clinicas de Porto Alegre under protocol number 110374 and Clinical Trial Register (NCT01450371). Subjects were informed about the study protocol and gave their informed written consent before their participation.

\section{Experimental Protocol}

Subjects were randomly allocated, using computer-based randomization with Graphpad StatMate ${ }^{\mathrm{TM}}$ software (La Jolla, CA, USA), in three groups: TENS, IES or Sham-stimulation group, with 48 hours rest between them and 72 hours after the first visit. In the first visit, subjects completed a health questionnaire and performed a maximal cardiopulmonary exercise test, as previously described. ${ }^{(17)}$ In the second, third and fourth visits, subjects were submitted to the randomized intervention during 30 minutes, applied in the region of cervical-thoracic ganglion (C7-T4), where $5 \times 5 \mathrm{~cm} 2$ adhesive electrodes (MultiStick ${ }^{\circledR}$, Axelgaard Manufacturing $\mathrm{CO}$, Ltd, Fallbrook, CA, USA) were placed on each side, about $3 \mathrm{~cm}$ to the right and left of midline vertebral process as described elsewhere. ${ }^{(2)}$ The TENS group received continuous flow, symmetrical and rectangular biphasic pulses using bipolar electrodes with two channels of TENS (TensMed 911 Device, Enraf-Nonius B.V., Rotterdan, Netherlands, GB 3004), with a frequency of $80 \mathrm{~Hz}$ and pulse width of $150 \mu \mathrm{s}$. For IES group, the carrier current was adjusted to $4000 \mathrm{~Hz}$, with AMF of the $80 \mathrm{~Hz}$, AMF variation of $25 \mathrm{~Hz}(25 \%$ of AMF) and slope of 1/5/1 (Endophasys nms.0501®, KLD Biosistemas, Amparo, SP, Brazil) $)^{(3)}$. Intensity was increased from zero to maximum sensitive threshold, which was the maximal individual level at which subjects did not report pain, discomfort or involuntary contraction. The same procedures were conducted in the sham-stimulation group, but the equipment did not provide any electrical current. ${ }^{(18)}$

\section{Muscle Metaboreflex Induction}

The muscle metaboreflex activity was evaluated as described elsewhere. ${ }^{(2,3,17)}$ Briefly, maximal voluntary contraction (MVC) of the dominant arm was initially determined with a handgrip dynamometer (Jamar® Hydraulic Hand Dynamometer, Sammons Preston CO, Bolingbrook, Illinois, USA). A static handgrip exercise was performed at $30 \%$ of MVC for $3 \mathrm{~min}$, immediately followed by post exercise circulatory occlusion with (PECO+) and without occlusion (PECO-) pressure measurement of the exercised arm, to promote selective induction of metaboreflex. Heart rate (HR) was measured by a heart rate monitor (POLAR model RS800, Kempele, Finland) and mean blood pressure (MBP) was measured in the non-dominant arm using a calibrated oscillometric automatic device (Dinamap 1846SX/P, Critikon, Tampa, Florida, USA). Calf blood flow (CBF) was measured by venous occlusion plethysmography (Hokanson, TL-400, Bellevue, USA). Calf vascular resistance (CVR) was calculated as MBP/CBF. (17)

Determination of MMA was performed by changes in systolic blood pressure which were measured and plotted against protocol time for both PECO+ and PECO-. The area under curve was estimated, and the calculated difference in the area between PECO+ and PECO- was regarded as MMA. All flow recordings were manually traced by an operator who was blinded to the intervention and time. Reproducibility of CBF measurements in our study group has been observed to be good with coefficients of variation of $5.7-5.9 \%$ for intra and inter-day measurements. ${ }^{(2,3)}$

\section{Heart Rate Variability}

Recordings obtained from the heart rate monitor were analyzed using intervals during the 9-min heart rate variability (HRV) data acquisition period, considering 256 heart beats, as described elsewhere (2). HRV in the frequency domain was calculated according to the Task Force of the European Society of Cardiology and the North American Society of Pacing and Electrophysiology (1996).. ${ }^{(19)}$ Power spectral component were reported using Fast Fourier Transform at LF and HF, expressed in normalized units. Temporal series from the tachogram, related to each selected segment were quantitatively evaluated considering the values for the HR, total and normalized powers (n.u) of low frequency (LF -0.04 to $0.15 \mathrm{~Hz}$ ) and high frequency (HF -0.15 to $0.40 \mathrm{~Hz}$ ) components of HRV and the sympatho-vagal index (LF/HF). Normalized units were obtained by dividing the power of a given component by the total power (from which VLF has been subtracted) and 
multiplying by $100 .{ }^{(20)}$ Analyses were performed with a personal computer using customized software (KUBIOS, Kuopio, Finland) as performed previously for our group. Artifacts were reviewed by visual inspection of the computer display. Only segments with 95\% pure sinus beats were included in the final analysis.

\section{Data Analysis}

Values are reported as means \pm SD. Twotailed unpaired $t$ tests were used to compare differences in baseline values between the groups. Differences in hemodynamic responses among TENS, IES and Control intervention during exercise and to PECO+/PECO- were compared by generalized estimating equation (GEE) models for repeated measures. Statistical significance was accepted when $p<0.05$. Data were analyzed using SPSS version 20.0 (SPSS, Chicago, IL, USA).

\section{RESULTS}

During the 4-month recruitment period, 18 subjects were screened. Seven subjects were excluded to not meeting inclusion criteria $(n=5)$ and declinate participate $(n=2)$. A total of 11 healthy subjects (age: $26 \pm 2.8$ years; height: 166 $\pm 4 \mathrm{~cm}$; body mass: $63 \pm 3 \mathrm{~kg}$ ) completed the study (Figure 1). The subjects had a maximal oxygen uptake of $38 \pm 0.3 \mathrm{ml} / \mathrm{kg} \cdot \mathrm{min}^{-1}$, assessed on previous ramp-incremental cycle ergometer exercise test. Initial maximal voluntary contraction (MVC) was $42 \pm 3 \mathrm{~N}$, assessed by handgrip dynamometer. ${ }^{(19)}$

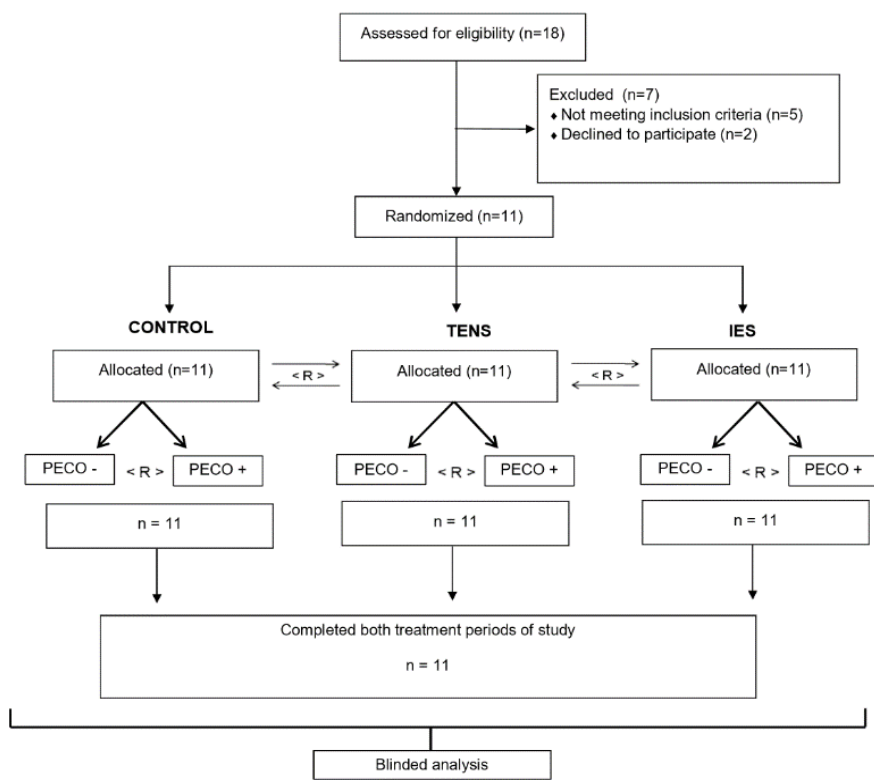

Figure 1: Flowchart of study

Note: CTL = Controls; TENS = transcutaneous electrical nerve stimulation; IES = interferential electrical stimulation. PECO = postexercise circulatory occlusion.

\section{Muscle Metaboreflex Activity}

Responses of MBP, HR, CBF and CVR data during TENS, IES and Sham-stimulation, at baseline, handgrip exercise, and during the recovery with and without immediate circulatory occlusion (PECO+ and PECO-, respectively) were shown in Figure 2. In all groups MBP was greater during exercise and the recovery period during PECO+ compared with PECO-. However, both modalities of electrical current evoked significant reduction on MBP at peak exercise compared with control condition (Shamstimulation $113 \pm 3$, TENS $103 \pm 2$ and IES $88 \pm$ $3 \mathrm{mmHg} ; \mathrm{p}<0.05)$ which was meaningly lower in IES group $(p<0.001)$ (Fig. 2A). HR did not present relevant changes between PECO groups in the baseline and exercise, but the IES reduced significantly HR during exercise, at peak exercise and recovery compared with Sham and TENS $(p<0.05)$ (Fig. 2A). Regarding CBF measure, TENS and IES increased value in baseline, exercise and recovery compared with Sham $(p<0.001)$, with a superior value on IES when comparing to TENS $(\mathrm{p}<0.05)$ (Fig. $2 \mathrm{~B})$. Unlike the $\mathrm{CBF}$, the CVR was significantly reduced in TENS and IES groups in all situations compared with Sham group in all situations $(p<0.05)$. Likewise, a higher reduction was found after application of IES $(p<0.001)$ (Fig. 2B).
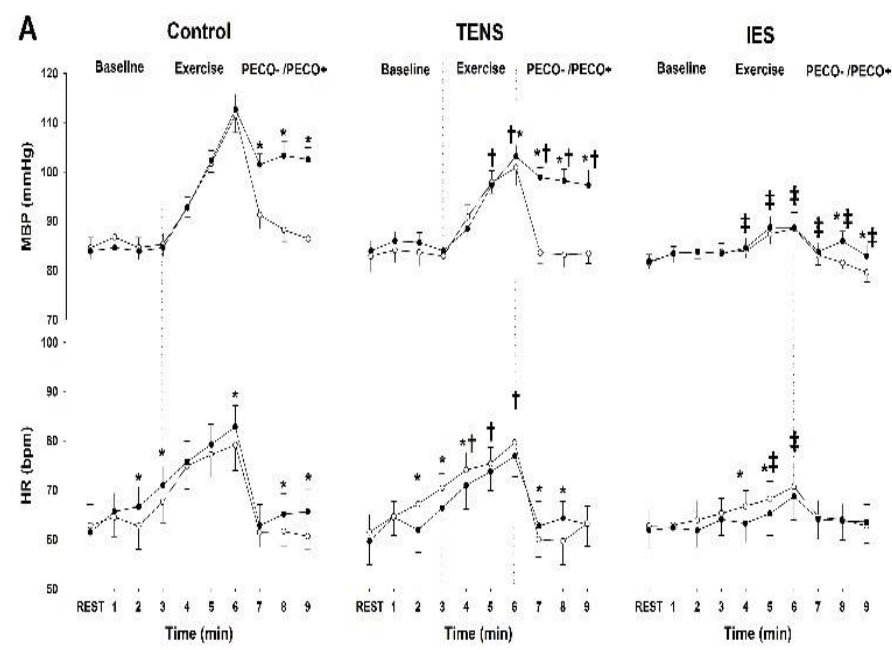

Figure 2: Mean blood pressure (MBP) and heart rate $(\mathrm{HR})$ in absolute values during the static hangrip exercise, and after exercise with (PECO+) and without (PECO-) circulatory occlusion in healthy subjects.

*Note: Statistical significance was accepted when $p<0.05$. * Generalized estimating equation (GEE) for repeated measures ( $p<$ 0.05): comparisons within intervention, PECO- vs. PECO+; † GEE for repeated measures $(P<0.05)$ : comparisons between interventions, Control vs. TENS vs. IES. 

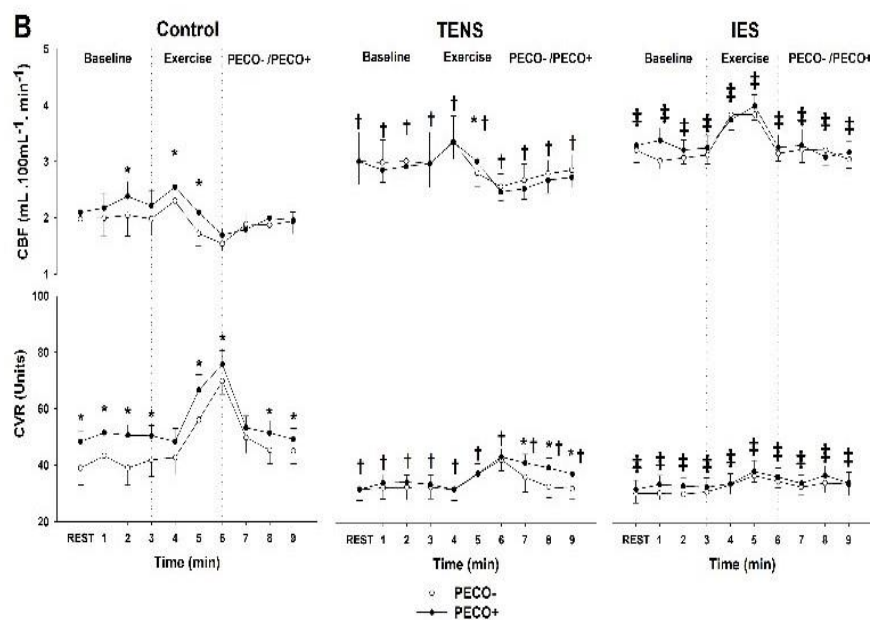

Figure 3. Calf blood flow (CBF), and calf vascular resistance $(C V R)$, in absolute values during the static handgrip exercise, and after exercise with (PECO+) and without (PECO-) circulatory occlusion in healthy subjects.

"Note: Statistical significance was accepted when $p<0.05$. * Generalized estimating equation (GEE) for repeated measures ( $p<$ 0.05): comparisons within intervention, PECO- vs. PECO+; † GEE for repeated measures: comparisons between interventions, Control vs. TENS vs. IES.

As a result of the measurements described above, MMA was significantly higher on Sham (26 \pm 5 units) compared with TENS and IES protocol (14 $\pm 4,9 \pm 2$ units; respectively) $(p<0.001)$. Additionally, IES resulted in greater reduction in MMA compared with other groups $(p<0.05)$ (Figure 3).

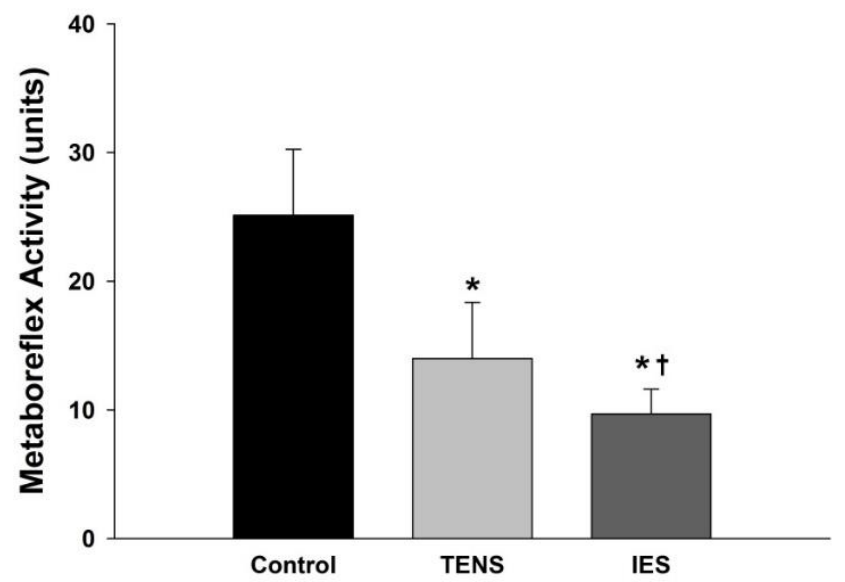

Figure 4: Estimated muscle metaboreflex control of calf vascular resistance, obtained by the subtraction of the area under the curve during circulatory occlusion from the control period, during Control, TENS or IES.

"Note: Generalized estimating equation (GEE) for repeated measures: $p<0.05$ for group, intervention and interaction effects. Multiple comparisons: * significantly different TENS and IES vs. Control; † significantly different IES vs. TENS.

\section{Heart Rate Variability}

Results for HRV parameters during PECOand PECO+ were shown in Figure 4. TENS and IES presented different responses when compared with Sham group $(p<0.001)$. LF and HF components were changed in both PECO- and PECO+ with TENS and IES $(p<0.001)$. Interestingly, these changes were more expressive during PECO+ with IES $(p<0.05)$. LF/HF ratio, which represent sympatho-vagal balance modulation, reduced significantly during PECO+ on TENS and IES compared with to Sham group $(p<0.01)$. Furthermore, IES resulted in higher reduction of LF/HF ratio during PECOand PECO+ $(p<0.05)$.

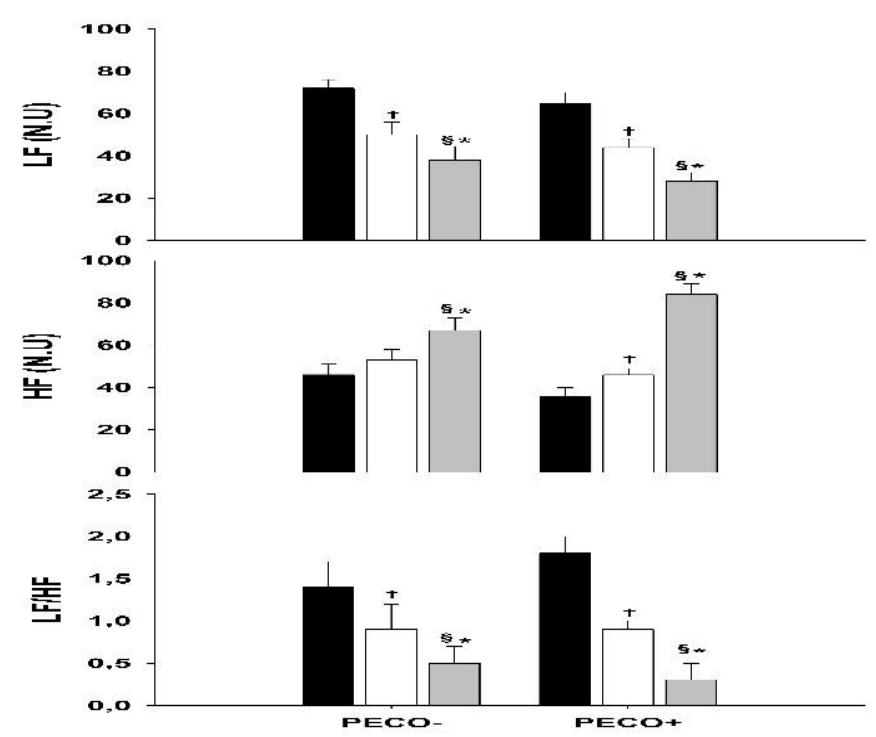

Figure 5: Heart rate variability indices of the frequency domain in Control, TENS and IES during PECO+ and PECO-. Black, white and gray bar (control, TENS, IES, respectively).

"Note: Generalized estimating equation (GEE) for repeated measures: $P<0.05$ for group, intervention and interaction effects. Multiple comparisons: * significantly different IES vs. Control; † significantly different TENS vs. Control; § significantly different IES vs.TENS $(\mathrm{p}<$ 0.01).

\section{DISCUSSION}

To our knowledge, this is the first randomized trial comparing IES and TENS effect, applied over the stellate ganglion region, over autonomic nervous system in healthy subjects. The main findings of this study are that IES have a superior effect compared to TENS to attenuate muscle metaboreflex activity by sympatheticvagal modulation in healthy subjects, as shown previously. ${ }^{(3)}$

At least in part, these findings may be underlied by a higher maximum total current and 
a more effective penetration of IES into deep tissues through kilohertz-carrier-frequency pulsed or sinusoidal currents to overcome the impedance of the skin. ${ }^{(16)}$ Differently, low frequency TENS studies produced a reduced skin conductance. ${ }^{(12,21)}$ If so, IES could generate larger alterations on muscle metaboreflex activity and sympathetic-mediated vasoconstriction, which may induce major local vasodilation during exercise.

In this study, IES applied on ganglion region was superior to TENS to improve CBF and reduce CVR, and, hence, decreasing muscle metaboreflex activity during exercise. A previous study by our group had already suggested that IES can generate peripheral vasodilatation in this population at peak exercise. ${ }^{(3)}$ Although it is the first study comparing IES and TENS electrical stimulation in healthy volunteers. Lamb found an increased arterial blood flow and skin perfusion during and after IES, ${ }^{(4)}$ and Ganne et al. demonstrated substantial vasodilatation in the upper limbs with the administration of IES to the brachial plexus region. ${ }^{(22)}$ Furthermore, the application of the electrical stimulation at ganglion level has resulted in a significant improvement of the blood flow in subjects with Raynold's Syndrome ${ }^{(23)}$ and Endarteritis Obliterans, ${ }^{(24)}$ which corroborates our findings.

In contrast, Nussbaum et $\mathrm{al}^{(25)}$ found no change in peripheral vasodilatation with the use of IES when applied to the cervical sympathetic chain and dorsal-lumbar region, regardless of the application site and intensity of the current. Other studies have reported that application of IES did not change peripheral blood flow and vascular resistance in healthy subjects during rest ${ }^{(1)}$ and did not increased cutaneous blood flow when applied quadriceps. ${ }^{(7)}$ This could be explained by different evaluation moments and local of electrical stimulation application, respectively.

We also found that the effect of IES was higher than TENS on the modulation of HRV, with increases of HF and decreases in LF component and LF/HF ratio during PECO+ or PECO-. Our group has already demonstrated that TENS results in HRV improvement, ${ }^{(2)}$ which is compatible with sympathetic nervous system activity reduction, perhaps by the CNS opioid release enhancement suggested by Campbell and Ditto. (21) Studies in chronic heart disease patients have reported that the application of TENS is linked to increase the baroreflex sensitivity, ${ }^{(13,14,26,27)}$ but none with ganglion application. Also, the sympatho-inhibitory effects of TENS also seems to have a beneficial effect on mean blood pressure. ${ }^{(28)}$ We found no studies evaluating HRV modulation after IES intervention. In this context, it could hypothetized that the modulating effects of IES and TENS on the opiod systems produced important systemic effects. For instance, low frequency TENS - as used in the present study - may activate $\delta$-opioid receptors in spinal cord(29) and rostral ventromedial medulla. ${ }^{(30)}$ These receptors are associated with vasoactive substances release such as endorphins which have dual effects in reducing pain and sympathetically-mediated vasoconstriction. ${ }^{(31,32)}$ However, we believed that TENS and IES at stellate ganglion evoked important effects on the opioid systems improving blood flow peripheral by vagal-tonus stimulation.

The present investigation has some important limitations which can drive the interest for future studies. Firstly, we did not evaluate muscle sympathetic nerve activity or catecholamines spill-over autonomic which could additionally provide supportive evidence for IES and TENS-induced reduction in sympathetic hyperactivity. Secondly, stellate ganglion blockade is related to an enhance of the cerebral blood flow. ${ }^{(33)}$ Thirdly, as described previously in our two papers, ${ }^{(2,3,32)}$ we used as control for application of TENS and IES electrodes at the same dorsal region. Fourthly, an additional limitation was did not attempt to directly assess endogenous opioid levels. On the other hand, as described in the literature, ${ }^{(21)}$ the usual method of assessing opioid levels by assay of plasma, may not be relevant for blood pressure.

\section{STUDY LIMITATIONS}

The likely contemporary stimulation of near structures which may affect the cardiovascular system should be considered and discussed.

\section{CONCLUSION}

In summary, the results of the present study demonstrate that ganglion neuromuscular electrical stimulation by TENS and IES was capable of attenuating the peripheral responses caused by muscle metaboreflex activity, maintaining peripheral blood flow and peripheral vascular resistance within the range of normality, with IES superiority. These findings contribute toward a better understanding of these types of therapies on these variables. The administration of these therapies may have an extremely positive impact on the treatment of patients with diseases that lead to an intolerance to exercise due exacerbation of muscle metaboreflex activity. 
Authors' contributions: Conceptualizatio: JZ, PJCV, GCJ, ALMW, MDS, AMGC, TZR, FVS and GRC. Data curation: JZ, PJCV, GCJ, ALMW, MDS, AMGC, TZR, FVS and GRC. Formal analysis: GCJ, FVS and GRC. Funding acquisition: GRC and GCJ. Investigation: JZ, PJCV, ALMW and MDS. Methodology: GSJ, AMGC, TZR, FVS and GCJ. Project administration: Gaspar R. Chiappa. Resources: GRC and GCJ .Chiappa.Validation: GRC and GCJ. Chiappa.Visualization: GRC and GCJ

Financial support: FIPE/HCPA and CNPq.

Conflict of interest: None of the authors have any potential conflict of interest related to the contents of this paper.

\section{REFERENCES}

1. Indergand HJ, Morgan BJ. Effect of interference current on forearm vascular resistance in asymptomatic humans. Physical therapy. 1995;75(4):306-12.

2. Vieira PJ, Ribeiro JP, Cipriano G, Jr., Umpierre D, Cahalin LP, Moraes RS, et al. Effect of transcutaneous electrical nerve stimulation on muscle metaboreflex in healthy young and older subjects. European journal of applied physiology. 2012;112(4):1327-34.

3. Valdez FS, Chiappa GR, Vieira PJ, Umpierre D, Ribeiro JP, Cipriano G, Jr. Interferential electrical stimulation improves peripheral vasodilatation in healthy individuals. Braz $\mathrm{J}$ Phys Ther. 2013;17(3):281-8.

4. Lamb SM, R. Does intereferential therapy affect blood flow? . Clin Rehab. 1994;8:2138.

5. ERP Nussbaum; Disenhaus L. The effects of interferential therapy on peripheral blood flow. Physiotherapy. 1990;76:803-7.

6. Cramp AF, Gilsenan C, Lowe AS, Walsh DM. The effect of high- and low-frequency transcutaneous electrical nerve stimulation upon cutaneous blood flow and skin temperature in healthy subjects. Clin Physiol. 2000;20(2):150-7.

7. Noble JG, Henderson G, Cramp AF, Walsh $D M$, Lowe AS. The effect of interferential therapy upon cutaneous blood flow in humans. Clin Physiol. 2000;20(1):2-7.

8. Hollman JE, Morgan, B. J. Effect of transcutaneous electrical nerve stimulation on the pressor response to static handgrip exercise. Physical therapy. 1997;77:28-36.

9. Indergand HJ, Morgan BJ. Effects of highfrequency transcutaneous electrical nerve stimulation on limb blood flow in healthy humans. Physical therapy. 1994;74(4):3617.
10. Sandberg ML, Sandberg MK, Dahl J. Blood flow changes in the trapezius muscle and overlying skin following transcutaneous electrical nerve stimulation. Physical therapy. 2007;87(8):1047-55.

11. Hallen K, Hrafnkelsdottir T, Jern S, Biber B, Mannheimer C, DuttaRoy S. Transcutaneous electrical nerve stimulation induces vasodilation in healthy controls but not in refractory angina patients. J Pain Symptom Manage.40(1):95-101.

12. Kaada B. Vasodilation induced by transcutaneous nerve stimulation in peripheral ischemia (Raynaud's phenomenon and diabetic polyneuropathy). Eur Heart J. 1982;3(4):303-14.

13. Mannheimer $\mathrm{C}$, Emanuelsson $\mathrm{H}$, Waagstein $F$. The effect of transcutaneous electrical nerve stimulation (TENS) on catecholamine metabolism during pacing-induced angina pectoris and the influence of naloxone. Pain. 1990;41(1):27-34.

14. Sanderson JE, Tomlinson B, Lau MS, So $\mathrm{KW}$, Cheung $\mathrm{AH}$, Critchley JA, et al. The effect of transcutaneous electrical nerve stimulation (TENS) on autonomic cardiovascular reflexes. Clin Auton Res. 1995;5(2):81-4.

15. Weil EH, Ruiz-Cerda JL, Eerdmans $\mathrm{PH}$, Janknegt RA, Van Kerrebroeck PE. Clinical results of sacral neuromodulation for chronic voiding dysfunction using unilateral sacral foramen electrodes. World J Urol. 1998;16(5):313-21.

16. Kloth L. Interference current. In: Nelson R, Currier, DP (eds), editor. Clinical Electrotherapy. Connecticut: Appleton \& Lange; 1991. p. 221-60.

17. Roseguini BT, Alves CN, Chiappa GR, Stein $\mathrm{R}$, Knorst MM, Ribeiro JP. Attenuation of muscle metaboreflex in chronic obstructive pulmonary disease. Med Sci Sports Exerc. 2008;40(1):9-14.

18. Cipriano G, Jr., de Camargo Carvalho AC, Bernardelli GF, Tayar Peres PA. Short-term transcutaneous electrical nerve stimulation after cardiac surgery: effect on pain, pulmonary function and electrical muscle activity. Interactive cardiovascular and thoracic surgery. 2008;7(4):539-43.

19. Electrophysiology TFotESoCtNASoP. Heart Rate Variability: Standards of Measurement, Physiological Interpretation, and Clinical Use. Circulation. 1996;93(5):1043-65.

20. Montano N, Porta A, Cogliati C, Costantino G, Tobaldini E, Casali KR, et al. Heart rate 
variability explored in the frequency domain: a tool to investigate the link between heart and behavior. Neurosci Biobehav Rev. 2009;33(2):71-80.

21. Campbell TS, Ditto B. Exaggeration of blood pressure-related hypoalgesia and reduction of blood pressure with low frequency transcutaneous electrical nerve stimulation. Psychophysiology. 2002;39(4):473-81.

22. Ganne JM. Interferential Therapy. Aust J Physiotherapy. 1976;22:101-10.

23. Schoeler H. Physical Block of Sympathetic Chain. Technik im der Medizin. 1972;1:16-8.

24. Nikolova-Troeva $L$. The modem electrotherapeutic methods in the therapy of endarteritis obliterans. Ther Gegenw. 1968;102:190-8.

25. Nussbaum ER, P.; Disenhaus, L. The Effects of Interferential Therapy on Peripheral Blood Flow. Physiotherapy. 1990;76(12):803-7.

26. Gademan MG, Sun Y, Han L, Valk VJ, Schalij MJ, van Exel HJ, et al. Rehabilitation: Periodic somatosensory stimulation increases arterial baroreflex sensitivity in chronic heart failure patients. Int $\mathrm{J}$ Cardiol. 2011;152(2):237-41.

27. Sherry JE, Oehrlein KM, Hegge KS, Morgan BJ. Effect of burst-mode transcutaneous electrical nerve stimulation on peripheral vascular resistance. Physical therapy. 2001;81(6):1183-91.

28. Kaada B, Flatheim E, Woie L. Low-frequency transcutaneous nerve stimulation in mild/moderate hypertension. Clin Physiol. 1991;11(2):161-8.

29. Sluka KA, Deacon M, Stibal A, Strissel S, Terpstra A. Spinal blockade of opioid receptors prevents the analgesia produced by TENS in arthritic rats. J Pharmacol Exp Ther. 1999;289(2):840-6.

30. Kalra A, Urban MO, Sluka KA. Blockade of opioid receptors in rostral ventral medulla prevents antihyperalgesia produced by transcutaneous electrical nerve stimulation (TENS). J Pharmacol Exp Ther. 2001;298(1):257-63.

31. Han JS, Chen XH, Sun SL, Xu XJ, Yuan Y, Yan SC, et al. Effect of low- and highfrequency TENS on Met-enkephalin-ArgPhe and dynorphin A immunoreactivity in human lumbar CSF. Pain. 1991;47(3):295-8.

32. Zadina JE, Hackler L, Ge LJ, Kastin AJ. A potent and selective endogenous agonist for the mu-opiate receptor. Nature. 1997;386(6624):499-502.
33. Ide $\mathrm{K}$, Boushel R, Sorensen HM, Fernandes A, Cai Y, Pott F, et al. Middle cerebral artery blood velocity during exercise with beta-1 adrenergic and unilateral stellate ganglion blockade in humans. Acta Physiol Scand. 2000;170(1):33-8. 